\title{
PLAN DE ROTACIÓN POR LA MODALIDAD DUAL PARA PRÁCTICAS PRE- PROFESIONALES.
}

M.Sc. Efraín Velasteguí López ${ }^{1}$, Ing. M.Sc. Eufemia Ramos ${ }^{2}$, Ing. Mg.Edwin Santamaría ${ }^{3}$ Dr. MBA. Walter Jiménez Silva ${ }^{4}$

\begin{abstract}
The Faculty of Administrative Sciences includes within the curriculum a program for students of Business Administration related to the implementation of preprofessional practices whose purpose is to link the academic training with practical work in companies, so that at the end of the process, with work experience.

Analytical research was carried out with the information obtained in the Department of Professional Practices of the organization of companies of the Faculty of Administrative Sciences in the period April to September 2016. In addition, we used the interview technique obtaining as a result that the $94 \%$ of the students made the practices in the areas of services and production and carried out activities in jobs related to the following areas: Production with a 32\%, 29\% human talent, logistics, $15 \%, 14 \%$ quality management and organization 10\%; these results were not encouraging for managers who request proposes a strategy to optimize resources. With the above information was made a diagnosis using the SWOT analysis came to the conclusion that requires a rotation plan under the dual mode with the intention of integrating the academy and the business environment.

The purpose of the pre-professional practices is to reinforce the theoretical learning with practical training and incorporate entrepreneurship education in productive sectors with strong growth of employability and programs that enhance skills, competencies and performance of future professional administrator.
\end{abstract}

The present study allows you to structure a creditable Integrator product through a rotation scheme for the future professional dual mode; whose implementation will develop skills, competencies and performance for the student to learn by doing, To ensure the construction and development of critical and creative thinking of the learner, through interactive processes, collaborative, participative and contextualized under the control of the guardian specialist, the student is called "apprentice", receive theoretical training at the Faculty of first to fifth semester and practice will be carried out in the company according to inter-institutional agreements. The development of the pre-professional practices will be carried out in a total of 400 hours in two levels of 200 hours each one according to the curriculum in force

\footnotetext{
${ }_{1}^{1}$ Universidad Técnica de Ambato Facultad de Administración, Ambato, Ecuador, le.velastegui@uta.edu.ec

2 Universidad Técnica de Ambato Facultad de Administración, Ambato, Ecuador, a.ramos@uta.edu.ec

${ }_{3}^{3}$ Universidad Técnica de Ambato Facultad de Administración, Ambato, Ecuador, e.santamaria@uta.edu.ec

${ }^{4}$ Universidad Técnica de Ambato Facultad de Administración, Ambato, Ecuador, w.jimenez@uta.edu.ec
} 
Keywords: Dual Mode, Rotation Plan, Guardian, Apprentice, Pre-Professional Practices

CÓDIGO UNESCO: Sistemas de Información 120318

\section{RESUMEN}

La Facultad de Ciencias Administrativas contempla dentro de la malla curricular un programa para los estudiantes de la Carrera de Administración de Empresas relacionado con el cumplimiento de prácticas pre-profesionales cuya finalidad es vincular la formación académica con trabajo práctico en las empresas, de modo que al concluir con el proceso, cuenten con experiencia laboral.

Se realizó una investigación analítica con la información obtenida en el Departamento de Prácticas Profesionales de la Carrera de Organización de Empresas de la Facultad de Ciencias Administrativas en el período abril-septiembre de 2016. Además, se utilizó la técnica de la entrevista obteniéndose como resultado que el $94 \%$ de los estudiantes realizaron las prácticas en los sectores de servicios y producción y ejecutaron actividades en puestos de trabajo relacionados a las áreas: producción con un $32 \%$, talento humano $29 \%$, logística $15 \%$, gestión de calidad $14 \%$ y organización $10 \%$; estos resultados no fueron nada alentadores para los Directivos quienes solicitan se proponga una estrategia que permita optimizar los recursos. Con la información antes mencionada se realizó un diagnóstico utilizamos el análisis FODA llegándose a concluir que se requiere un plan de rotación bajo la modalidad dual con la intención de integrar la academia y el entorno empresarial.

El propósito de las prácticas pre-profesionales es reforzar el aprendizaje teórico con capacitación práctica e incorporar la educación empresarial en sectores productivos con gran crecimiento de empleabilidad y programas que potencien habilidades, competencias y el desempeño del futuro administrador profesional.

El presente estudio permite estructurar un producto integrador acreditable a través de un Plan de Rotación para el futuro profesional por modalidad dual; cuya aplicación permitirá desarrollar habilidades, competencias y desempeños para que el estudiante aprenda haciendo, lo que garantizará la construcción y desarrollo del pensamiento crítico y creativo del aprendiz, a través de procesos interactivos, colaborativo, participativo y contextualizado bajo el control del tutor especialista, el alumno se denomina "aprendiz", recibe la formación teórica en la Facultad de primero a quinto semestre y la práctica lo realizará en la empresa de acuerdo a convenios interinstitucionales. El desarrollo de las prácticas pre-profesionales se realizará en un total de 400 horas en dos niveles de 200 horas cada uno de acuerdo a la malla curricular vigente

Palabras Clave: Modalidad dual, plan de rotación, tutor, aprendiz, practicas pre profesionales 


\section{INTRODUCCIÓN}

Analizados los resultados de prácticas pre-profesionales de la Carrera de Organización de Empresas correspondiente al período académico abril-septiembre de 2016 se determina que han participado 140 estudiantes pertenecientes a: séptimo, octavo, noveno, décimo y unos pocos que ya terminaron la malla. Los señores docentes tutores asignados para el seguimiento controlaron a través de visitas in situ y vía telefónica a las diferentes empresas públicas y privadas. Además, aplicaron cinco formatos estandarizados por la DIVISO y la legalización de convenios. Se evidencia que el $30 \%$ de los estudiantes se demoran, en promedio de uno a dos meses para presentar el informe final de prácticas, mismo que es requisito para validar el desempeño laboral. El estudiante entrega un archivo digital con todos los formatos validados y sellados en un $\mathrm{CD}$ por duplicado.

Se revisó el proceso, hemos encontrado en forma general que: el estudiante no puede ser juez y parte al mismo tiempo, por lo que los informes no deben ser generados por los estudiantes, sino por los tutores específicos y los supervisores en relación al cumplimiento de actividades de prácticas pre-profesionales con el fin de evidenciar los procesos de aprendizaje.

Para solucionar el problema: ¿Cómo afecta en la formación profesional de los egresados, la asignación de actividades prácticas en la gestión empresarial? presentamos como alternativa el plan de rotación con modalidad dual, con la participación del tutor general, tutores específicos y supervisor de prácticas pre-profesionales como un sistema de gestión alternativa con la intervención de la academia y la empresa y fortalecer la calidad educativa.

El Plan de rotación y la participación del tutor general, supervisores y tutores específicos permitirá en la mayoría de los casos, que en los convenios se especifique los puestos de trabajo y las funciones en base a necesidades reales.

Además, las prácticas pre-profesionales y las pasantías son procesos de gestión que impulsan la inserción laboral, la aprobación del presente proyecto es una estrategia para fortalecer e implementar mejoras continuas en los procesos vigentes.

Por otra parte, los estudiantes que concluyan las prácticas pre-profesionales, conocerán la problemática de las empresas in situ y esa información servirá para implementar temas de investigación que apoyen a la matriz productiva de la Provincia.

\section{SITUACIÓN ACTUAL DE LAS PRACTICAS PRE PROFESIONALES EN LA CARRERA DE ORGANIZACIÓN DE EMPRESAS}

Mediante resolución FCA-CD-P-0027-2016 de 22 de enero de 2016 se aprueba el Instructivo de Prácticas Pre-profesionales denominado "Seguimiento de Prácticas de Formación Académica", mismo que consta como alcance a la resolución FCA-CD-P-9962015 de julio 2 de 2015 y que indica la inclusión de nuevos formatos detallados como: Oficio de aceptación de la empresa/Institución, solicitud para realizar las prácticas de formación académica, certificado otorgado por la empresa, informe técnico de prácticas de formación académica, registro de asistencia, matriz de seguimiento, evaluación práctica de 
formación académica empresarial, convenio específico (documento que reemplaza a las cartas de compromiso).

En el período abril - septiembre 2016, los estudiantes que luego de cumplir con al menos 150 créditos y que realizaron las prácticas pre profesionales, el 71\% lo hicieron en el sector Servicios, seguido del 23\% en Producción, 5\% en el Comercio y el $1 \%$ en el sector Manufactura, es decir que el 94\% de nuestros estudiantes han cumplido sus prácticas preprofesionales en los sectores de Servicios y Producción

Las actividades que realizaron los estudiantes durante las prácticas agrupadas en unidades y/o departamentos: $32 \%$ en actividades relacionadas con Producción (P), 29\% con Talento Humano (TH), 15\% con Logística o Bodega (L),14 \% con Gestión de Calidad (GC) y 10\% con Organización de Empresas (OE).

Tomando como base las principales actividades que el estudiante cumple durante las prácticas y considerando que son insumos para elaboración de proyectos de investigación; se establecen los siguientes Programas de Investigación: Gestión del Cliente, Sistematización, Talento Humano, Normas de Calidad

Con la información estadística, se analizó situación actual y realizamos el diagnostico, a través del FODA para conocer la situación actual de las Prácticas pre-profesionales (Tabla 4) y se llegó a la siguiente conclusión:

Se requiere elaborar una propuesta de un plan de rotación de prácticas pre-profesionales bajo la modalidad dual en correlación a: líneas de investigación de la Carrera de Organización de Empresas, perfil profesional y campo ocupacional en las diferentes áreas de la empresa, bajo la supervisión de tutores especialistas y supervisores académicos y empresariales

\section{PLAN DE ROTACIÓN MODALIDAD DUAL}

\section{Modalidad Dual}

Según la bibliografía consultada (Chile, 2012), (E.universitario, 2014), (Senescyt, 2015), (Teruel, 2016) y (Chicaiza \& Parra, 2016) la modalidad dual armoniza el aprendizaje de las aulas con el que se efectúa en la empresa, los aprendices (estudiantes) se formarán en dos entornos: académico y laboral; esto rompe con las formas tradicionales de la enseñanza y contribuye a la sociedad con la generación de empleo de calidad y preparación práctica para los jóvenes profesionales del futuro. Los estudiantes aplicarán principios de autonomía, responsabilidad y autogestión al enfrentarse al campo laboral y académico.

La modalidad dual permite una transformación de los estudiantes y docentes, lo que permite formalizar por medio de dos entornos, académico y laboral. Este desarrollo rompe con la estructura tradicional de la enseñanza. Gracias a esta modalidad los estudiantes al enfrentarse a los diferentes ámbitos tanto académicos como laborales tienen una visión clara del entorno. El sistema de aprendizaje por la modalidad dual es la oportunidad que 
tiene el sector productivo para contar con el talento humano calificado en corto plazo y con altas cualidades técnicas.

\section{Plan de rotación modalidad dual}

Según (Procel, 2012), (Castillo, 2015), (Medina, 2006), (Valverde, 2006), (Rojas, 2002), el plan de rotación de la modalidad dual es el marco de formación que se elabora en base a los objetivos empresariales o de enseñanza, establece según los niveles de formación y conocimientos del aprendiz, las actividades de mejora que desarrollará en la empresa, el plan de rotación produce entornos educativos responsables y productivos y se realiza bajo la supervisión de la entidad formadora y de las Instituciones de Educación Superior, esto hace que el aprendiz aumente su desempeño académico y ejecute actividades prácticas y reales, acorde a su formación.

Se realiza en dos entornos de aprendizaje:

- Los entornos educativos: son entidades formadoras responsables de la formación profesional del estudiante y que le corresponde acompañar en el proceso de formación, con tutores especialistas y con experiencia en la modalidad dual. La academia es el escenario de aprendizaje para la transferencia de conocimientos y uso de tecnología aprobados en la malla curricular de acuerdo a las disposiciones vigentes en la Ley de Educación Superior.

- Los entornos empresariales: son organizaciones que permiten bajo convenio, la aplicación y experimentación de los aprendizajes en procesos de producción o de servicios reales, o mediante plataformas virtuales, o en ambientes que emulen las características propias de la gestión empresarial.

En el 2016, en Ecuador según lo estipulado en el Art. 3, literal a) de la Normativa para Carreras y Programas en modalidad dual aprobado por el Consejo Educación Superior (CES), indica que la modalidad dual es: "... el componente de aprendizaje teórico se desarrollará en el entorno educativo institucional, bajo la responsabilidad de las instituciones de educación superior; mientras que los componentes teórico-prácticos se desarrollarán en entornos de producción o de servicios reales, virtuales o simulados..." por tanto, el aprendizaje del estudiante se produce tanto en entorno institucional como en el sector productivo. Las actividades laborales reales, virtuales y simuladas constituyen el eje organizador del currículo a través del plan de rotación. Su desarrollo u operacionalización es la gestión del aprendizaje práctico con tutorías profesionales y académicas con inserción del estudiante en el contexto en base a la planificación que consta en el plan de rotación define los puestos de trabajo que requiere la organización que da apertura para el convenio con la modalidad dual".

Existen muy pocas universidades que dentro de sus carreras tiene modalidad dual por su compleja estructura de funcionamiento entre estas tenemos: La Universidad Católica de Santiago de Guayaquil, Escuela Superior Politécnica de Chimborazo, Universidad de Cuenca, Universidad San Francisco de Quito, Universidad de las Fuerza Armadas, Universidad Espíritu Santo entre otras.

Integración de Áreas Específicas del Plan de Rotación con los Programas de Investigación y Líneas de Investigación 
En la Figura siguiente, se observa como funcionaría el Plan de Rotación en forma integral:

\section{MODALIDAD DUAL PLAN DE ROTACIÓN}



Elaborado por: Comisión Rediseño. 


\section{Figura 2}

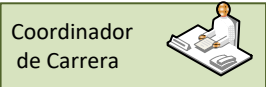

Coordinador de Vinculación


Asignaturas

Integradoras

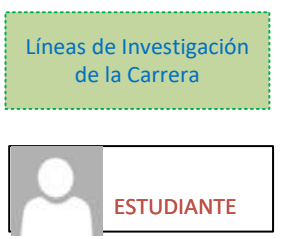

Áreas del Plan de

rotación

PUESTOS DE TRABAJO

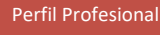

Perfil Ocupacional

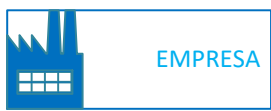

\section{NOMENCLATURA}

Recibe el informe final de las practicas pre profesionales y administra los resultados

Recibe el informe final de las practicas pre profesionales

Sustentar la validez de los convenios Públicas y Privadas. Elabora el plan de rotacion y asigana a tutores especificos, supervisores, y empresas con la carga de trabajo de acuerdo al distributivo para remitir al cordinador de carrera y ser aprobado. Ademas, facilita la cooperación, diálogo y solución de los problemas que se registren entre la Academia y la Empresa, generando los resultados a traves de informes pertinentes.

Apoya y evalúa los tutores específicos. Determinar necesidades de las empresas públicas y privadas, Colabora con el tutor genera para la planificación y ejecución del plan de rotación. Ejecuta la cooperación, diálogo y solución de los problemas que se registren entre la Academia y la Empresa.

Acompañar en el proceso de formación a los aprendices en el componente de prácticas pre-profesionales, facilitando el desarrollo del aprendizaje, trabajo, creativo y experiencia en la aplicación de conocimientos en la empresa, de acuerdo a los parámetros determinados en las áreas específicas Remite, controla y reporta informes pertinentes al supervisor y al tutor general, participa en la planificación del Plan de Rotación.

Asignatura Integradora Sexto: Gestión de la Calidad Total (Planificación Financiera, Aplicación de la mercadotecnia, Aplicación de investigación Operativa II, gestión del derecho Tributario, Aplicación de sistemas de información en la Empresa Asignatura Integradora Séptimo: Planificación Estratégica (planificación financiera Il, desarrollo de habilidades gerenciales, Instrumentación de la psicología en la industria, gestión de Publicidad y promoción, gestión tributaria, aplicación de modelos de negocias Ebusines en la Empresa

Las lineas de investigacion son la generacion de la formacion integral definiendoce como un espacio estructurado de problemas $u$ objeticvos de investigacion dentro de un campo del conocimiemto permitiendo realizar procesos practicos e innovadores

El alumno se denomina "aprendiz", recibe la formación teórica en la Facultad de primero a sexto semestre y la práctica lo realizará en la empresa de acuerdo al convenio.

El Plan de rotacion permite al aprendiz rotar o alternar de acuerdo a una planificacion estructurada en las distintas areas de desempeño asignadas a los aprendices de sexto y séptimo

Funciones especificas capacidades habilidades definidas jerárquicamente por la empresa

1.Sólida formación y dominio de conocimientos científicos, tecnológicos y empresariales para la administración de recursos. 2.Capaz de diagnosticar y proponer alternativas de solución a situaciones de optimización de recursos.

3.Capacidad emprendedora para iniciar nuevos proyectos, y buscar y percibir oportunidades.

4.Capaz de administrar eficientemente los recursos de la organización, creando un clima laboral óptimo.

5.Capacidad de índole ejecutiva y estratégica: Toma de decisiones, fijación de objetivos, determinación de políticas, elaboración de planes, organización del trabajo e identificación de problemas.

6.Capacidad de índole administrativa y táctica: Control de tiempo, costo y eficiencia, solución de problemas, determinación de presupuestos y programas, y elaboración de informes.

7.Capacidad de liderazgo y comando: Capacidad de mantener una comunicación eficiente y provocar la motivación efectiva de su personal.

Al cumplir con la etapa de formación académica, el egresado de la carrera de organización de empresas poseerá características específicas que le permitan desempeñar actividades en el área laboral, en la investigación, en la consultoría de empresas, en la gestión de empresas, de entes públicos, privados o entidades sin fines de lucro, en las áreas de aprovisionamiento, producción, finanzas o investigación y desarrollo, como docente universitario con enorme fortaleza en las áreas de administración, finanzas y recursos humanos, como administrador de riesgos empresariales y financieros, como asesor de inversiones públicas y privadas, como empresario independiente; con cualidades de:

Las empresas ofrecerán plazas formativas en base al plan de rotación propuesto por los tutores específicos a través de un convenio de formación para los estudiantes que soliciten ser aprendices y cumplan con 150 créditos como requisito

Elaborado por: Comisión Rediseño. 
En la Tabla 5, se puede apreciar la integración entre las líneas de investigación, los programas de investigación y las áreas del Plan de Rotación, esto garantiza que los aprendizajes del estudiante son los que realmente la academia y la empresa requieren.

\section{Tabla 5}

Integración de Áreas Específicas del Plan de Rotación con los Programas de Investigación y Líneas de Investigación.

\begin{tabular}{|c|c|c|c|}
\hline Carrera & $\begin{array}{c}\text { Línea de } \\
\text { investigación }\end{array}$ & $\begin{array}{l}\text { Programa de } \\
\text { investigación }\end{array}$ & $\begin{array}{l}\text { Áreas del plan de } \\
\text { rotación }\end{array}$ \\
\hline \multirow[t]{8}{*}{$\begin{array}{l}\text { Organización } \\
\text { de empresas }\end{array}$} & \multirow[t]{2}{*}{$\begin{array}{l}\text { Desarrollo } \\
\text { empresarial } \\
\text { territorial }\end{array}$} & $\begin{array}{c}\text { Sistemas productivos } \\
\text { locales }\end{array}$ & Producción \\
\hline & & $\begin{array}{c}\text { Empleo y mercado de } \\
\text { trabajo }\end{array}$ & $\begin{array}{c}\text { Gestión del talento } \\
\text { humano }\end{array}$ \\
\hline & \multirow[t]{2}{*}{$\begin{array}{l}\text { Productividad y } \\
\text { competitividad } \\
\text { empresarial }\end{array}$} & Gestión Organizacional & $\begin{array}{c}\text { Estructura } \\
\text { organizacional y } \\
\text { funcionamiento } \\
\text { administrativo de la } \\
\text { organización }\end{array}$ \\
\hline & & Gestión de la Calidad & Gestión de calidad \\
\hline & Emprendimiento & Emprendimiento & Investigación de \\
\hline & y economía & & desarrollo de nuevos \\
\hline & \multirow[t]{2}{*}{$\begin{array}{l}\text { popular } \\
\text { solidaria }\end{array}$} & & $\begin{array}{c}\text { productos y/o } \\
\text { servicios. }\end{array}$ \\
\hline & & $\begin{array}{c}\text { Economía popular y } \\
\text { solidaria }\end{array}$ & $\begin{array}{c}\text { Gestión Financiera } \\
\text { (Cooperativas) }\end{array}$ \\
\hline
\end{tabular}

Fuente: líneas de investigación vigente de la Facultad de Ciencias Administrativas Elaborado por: Comisión Rediseño

\section{Diagrama de flujo para la validación del Plan de Rotación}

En la Figura 3, se esquematiza el proceso que debería seguirse para validar el Plan de Rotación 
Empresa

TUTOR

TUTOR

ESTUDIANTE

SUPERVISOR

GENERAL

ESPECIFICO
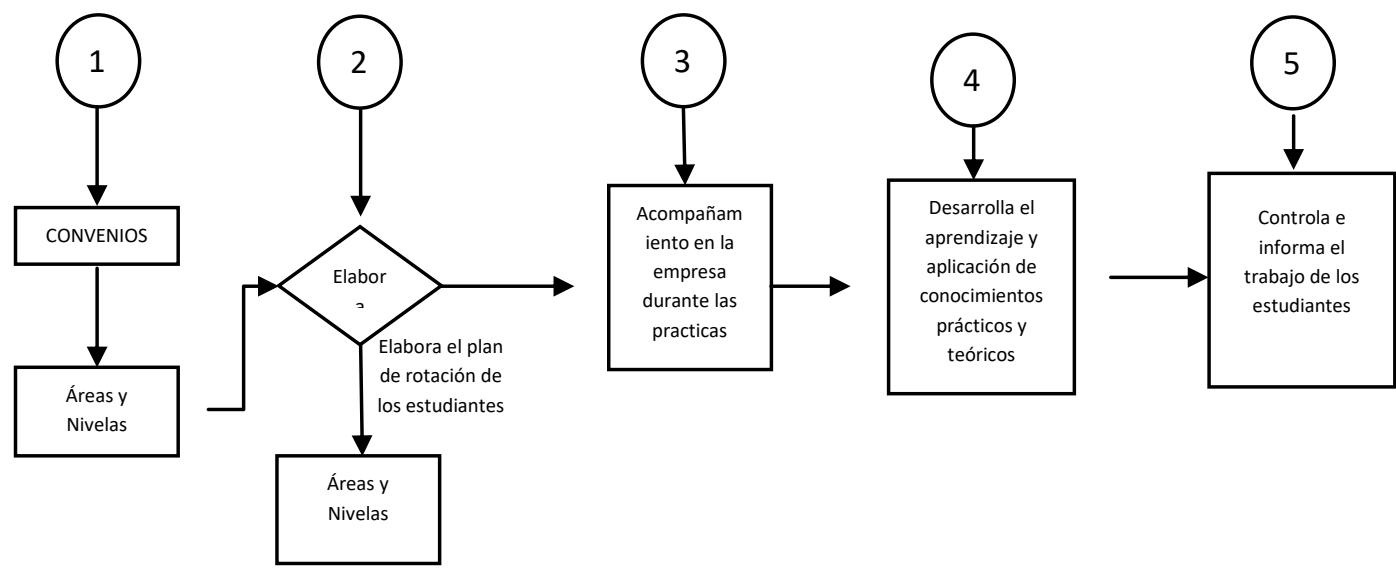

Figura 3. Validación del Plan de Rotación.

Elaborado por: Grupo de Diseño Curricular

\section{Descripción de procedimientos del Plan de Rotación y puestos de trabajo por áreas específicas en la Modalidad Dual}

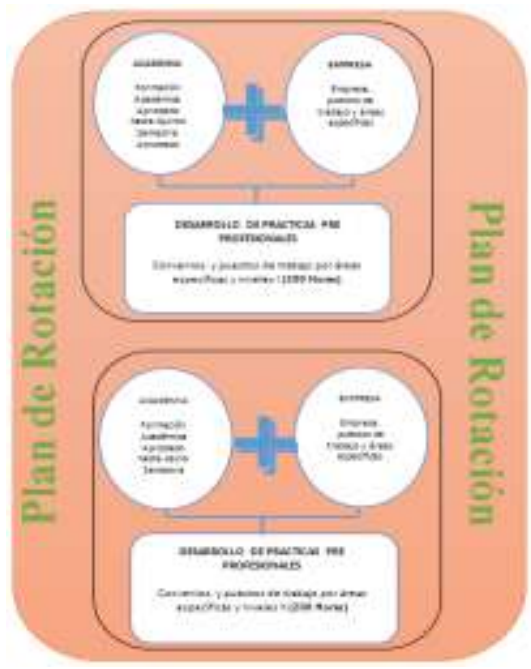

Figura 4. Plan de rotación y puestos de trabajo por áreas específicas. Elaborado por: Comisión de Diseño Curricular

En el ámbito empresarial: el responsable de la organización académica, denominado tutor general coordinará las acciones para que el tutor específico y el supervisor de la Carrera de Organización de Empresas y el Coordinador de la empresa identifique las áreas de trabajo potenciales para la formación del aprendiz para que definan los puestos de trabajo en los cuales se incorporarán a los aprendices y se defina el Plan de Rotación Específico. 
En el ámbito académico, el Coordinador de la Carrera en base al Convenio específico es responsable de verificar la correspondencia entre la formación que se determina en la empresa y las especificaciones institucionales; además, será quien proponga las acciones complementarias que garanticen el cumplimiento total de la normativa y el plan de rotación, disciplinares y profesionales, en apego al perfil profesional, perfil ocupacional, líneas de investigación y áreas específicas del futuro profesional.

\section{Proceso de Prácticas Pre profesionales}

Para que fluya adecuadamente el proceso mencionado, se ha identificado las siguientes etapas (Figura 3.).

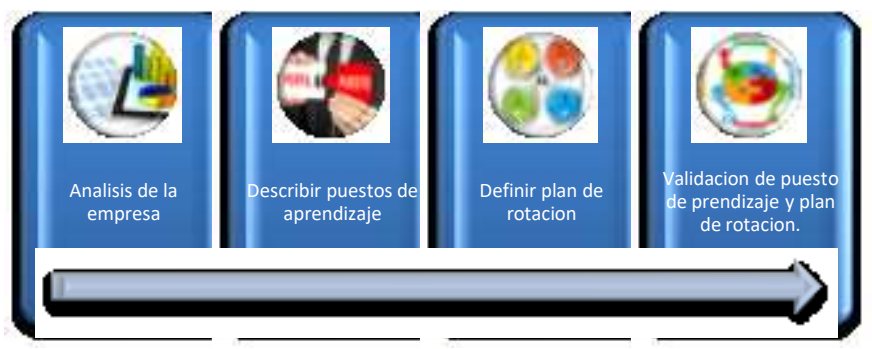

Figura 5. Proceso de Prácticas Pre profesionales, Elaborado por: Comisión de Rediseño Curricular.

\section{Análisis de la empresa}

El análisis de la empresa es una etapa en la que se colecta la información de diferentes fuentes, con la finalidad de definir el conjunto de comportamientos observables que determinan el desempeño en el puesto concreto, el cual será el insumo para definir el puesto de aprendizaje.

El análisis de la empresa se realiza los pasos que se señalan en la Figura 6:


Figura 6. Análisis de la empresa en tres pasos. Elaborado por: Grupo de Diseño Curricular 


\section{a) Identificar las áreas de la empresa}

Habrá que delimitar las áreas de la empresa, donde el educando/aprendiz, pueda formarse de acuerdo con la carrera de la Facultad; considerando que en la empresa tiene lugar un encadenamiento de valor que resulta en un fin determinado, en el cual se pueden identificar funciones y etapas, en correspondencia con áreas de la empresa.

- Conocer el perfil del Profesional de acuerdo a las líneas de investigación de la carrera y los puestos de trabajo.

- Identificar procesos diferenciados ya sea por los productos que se esperan en cada proceso, o por los conocimientos para llevar acabo los citados procesos, o por el uso de las tecnologías, maquinaria y equipo, o bien, por los insumos requeridos. (área)

- Identificar las áreas de la empresa que corresponden con el perfil del egresado

\section{b) Identificar puestos de trabajo}

Identificar en cada área productiva las funciones diferenciadas, o sub-áreas y los puestos de trabajo en los que está organizada, es decir el conjunto de actividades realizables por una sola persona.

- Identificar los procesos parciales o sub-áreas de que consta cada área de la empresa.

- Identificar los puestos de trabajo involucrados en el funcionamiento de la subárea.

- Verificar que los puestos de trabajo estén relacionados con el perfil profesional.

\section{c) Acopio de información}

Comprende buscar o generar información relativa al puesto de trabajo, para su descripción.

- Identificar documentos de referencia del puesto de trabajo tales como manuales de organización, manuales de procesos de calidad y descripción de puestos de trabajo.

- Identificar trabajadores en activo con amplia experiencia y conocimientos de los puestos de trabajo que puedan proporcionar la información.

\section{d) Los objetivos y las actividades del puesto de trabajo}

Supone información que pueda concretar una seria de actividades y la forma en las que esta debe ejecutarse.

- Identificar la razón de ser del puesto de trabajo.

- Definir los objetivos a cubrir. 
- Desagregar las actividades en congruencia con los objetivos.

- Detallar los comportamientos esperados.

\section{e) Definir conocimientos requeridos}

Esta etapa permitirá contar con una visión de los diferentes tipos de saberes que se ponen en juego durante la realización de las actividades del puesto de aprendizaje.

- Indicar los conocimientos necesarios para desempeñar las actividades.

- Indicar las habilidades y destrezas requeridas para el desempeño de las actividades

- Indicar las actitudes específicas para desempeñar las actividades.

\section{f) Detallar especificaciones}

Las especificaciones, es información adicional requerida para el desempeño en el puesto de trabajo:

- Definir los requerimientos en términos de cualidades de una persona que puede cubrir el puesto de trabajo.

- Definir requerimientos, administrativos, procedimentales, laborales u otro necesario durante la ejecución de las actividades

- Definir el tiempo que se puede desarrollar la función del puesto del trabajo

\section{Describir puestos de aprendizaje}

El puesto de aprendizaje es un instrumento que detalla las actividades que un educando/aprendiz realiza en un área específica de la empresa, en un tiempo determinado; la empresa responsable de generar y registrar los puestos de aprendizaje, con el apoyo del representante de la empresa, del tutor general y especifico en coordinación con el supervisor

El puesto de aprendizaje es resultado de una investigación de las actividades que se desarrollan en los puestos de trabajo de la empresa; se estructura a partir de una situación laboral, la cual será asimilada por el aprendiz.

Para definir los puestos de aprendizaje será necesario indicar los objetivos de aprendizaje y detallar los comportamientos esperados a manera de listado de funciones y tareas que adopten el nivel de dominio y grado de responsabilidad que el educando/aprendiz demostrará durante el proceso de formación; el aprendizaje en la empresa es un proceso progresivo que requiere de la instrucción y supervisión permanente del tutor especifico asignado.

Al contar con el puesto de aprendizaje, esto favorecerá la selección del estudiante idóneo. Para ello se diseñará un formato que permita estructurar y organizar el puesto 
de aprendizaje, mismo que podrá requisarse a partir de los hallazgos del análisis de la empresa.

Tabla 6

Puestos de trabajo

\begin{tabular}{|c|c|}
\hline Áreas de trabajo & Puestos (Asistente de.) \\
\hline Producción & Jefe de producción, supervisor, \\
\hline Gestión del talento humano & $\begin{array}{l}\text { Administrador, analista, asistente, de } \\
\text { Talento Humano }\end{array}$ \\
\hline $\begin{array}{l}\text { Estructura organizacional } \\
\text { fundamento administrativo de una } \\
\text { organización }\end{array}$ & Director, jefe, consultor, asesor \\
\hline Gestión de la Calidad & $\begin{array}{l}\text { Auditor, especialista, coordinador, líder de } \\
\text { procesos }\end{array}$ \\
\hline $\begin{array}{l}\text { Investigación y desarrollo de nuevos } \\
\text { productos y/o servicios }\end{array}$ & Jefe de planeación, director \\
\hline
\end{tabular}

Elaborado por: Grupo de Diseño Curricular

Para ejecutar el plan de rotación es importante incluir la asignatura integradora y el puesto de trabajo (asistente de todos los niveles jerárquico de la organización) que será en relación a las áreas específicas de la academia, líneas de investigación de la carrera, perfil profesional y perfil ocupacional.

\section{Definir plan de rotación}

El puesto de aprendizaje permitirá el diseño de un plan de rotación en la empresa, este último, como su nombre lo indica, establece la ruta de la formación del aprendiz, a cumplir en tantos puestos de aprendizaje sean necesarios.

El plan de rotación corresponde a la secuencia en la que organiza el proceso de formación en la empresa, se establece a partir del orden asignado a los puestos de aprendizaje para ser desempeñados por el aprendiz en un área específica.

Una vez que se cuente con el plan de rotación será necesaria su validación por la contra parte académica. 


\section{Validación del plan de rotación y del puesto de aprendizaje}

La validación de puesto de trabajo y plan de rotación es una tarea responsabilidad del gestor de vinculación apoyado por el tutor, consiste en establecer la correspondencia entre los aprendizajes definidos por la empresa.



Figura 8 Validación del Plan de Rotación y Puestos de aprendizaje. Elaborado por: Grupo de Diseño Curricular

Para lo anterior será necesario contar con los siguientes insumos: perfil del egreso, mapa curricular y programa de estudios de la carrera correspondiente; así como el plan de rotación y los puestos de aprendizaje, los cuales serán proporcionados por el operador del centro empresarial.

\section{a) Identificar Resultados de Aprendizaje}

Para poder identificar los resultados de aprendizaje, se deberán analizar el programa de estudio de cada módulo, cuya programática describe tanto los aprendizajes esperados por el estudiante, como la acción de enseñanza, aprendizaje y evaluación que conducirá a lograr dichos aprendizajes.

En la Carrera de Organización de Empresas, la Asignatura Integradora de sexto semestre es Gestión de la Calidad Total. Se observa todas las materias que la tributan, es decir podemos ver claramente los conocimientos que tiene el estudiante antes de realizar la práctica pre profesional.

En séptimo semestre la asignatura integradora es Planificación Estratégica. Observamos las materias que la tributan, esta tabla nos permite determinar los conocimientos que tiene el estudiante al llegar a este nivel de estudio. 
Tomando como base la asignatura de Gestión de la Calidad Total de sexto semestre (asignatura integradora), interrelacionándola con las líneas de investigación y el perfil profesional, se planificará los resultados de aprendizaje que exige el Plan de Rotación, esta actividad se desarrollará con la participación del tutor especialista.

Los resultados de aprendizaje, servirán como evidencia del desempeño del aprendiz en el puesto de trabajo asignado. Es necesario indicar que los puestos de trabajo del aprendiz, asignado por la empresa y el programa de estudio, en cualquier nivel jerárquico tendrá el grado de asistente. (Ejemplo: Asistente del Especialista en Gestión de la Calidad).

En la figura 9, se puede ver la asignación de Resultados de Aprendizaje para el puesto del ejemplo anterior y tomando como base la Asignatura Gestión de la Calidad Total

\section{Asistente del especialista en Gestión de la Calidad (Puesto trabajo)}

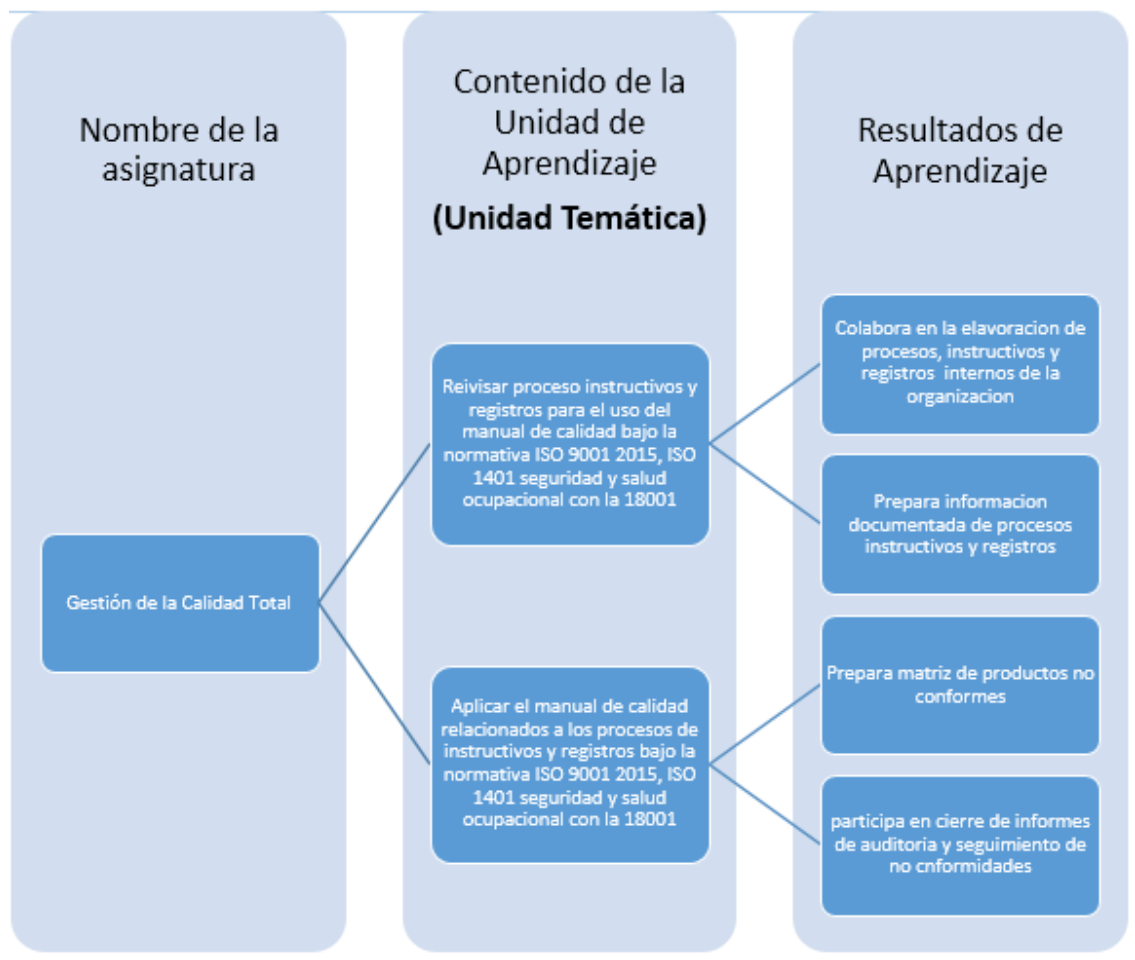

Figura 9. Asistente del especialista en Gestión de la Calidad (Puesto trabajo), Asignatura: Gestión de la Calidad Total. Elaborado por: Comisión de Re diseño Curricular

Continuando con el procedimiento, se requiere contar con el total de resultados de aprendizaje de los módulos de formación básica y profesional, que conforman la carrera.

Para lo anterior, será importante listar los resultados de aprendizaje localizados. 


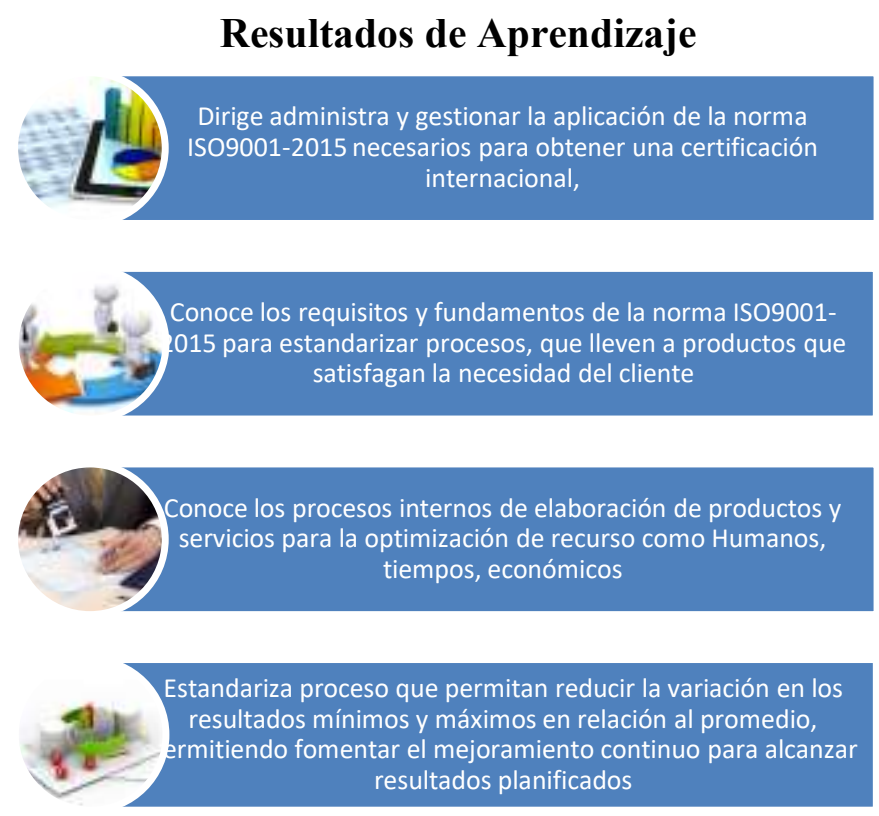

Figura 10. Resultados de aprendizaje. Elaborado por: Comisión de Diseño Curricular

Este listado constituye el elemento de referencia, contra el cual se cotejan los objetivos de aprendizaje especificados en el plan de rotación y puestos de aprendizaje de la empresa.

\section{b) Identificar los Objetivos de Aprendizaje}

Los objetivos de aprendizaje fueron determinados por la empresa en cada puesto de aprendizaje, para indicar los resultados parciales de formación que el educando/aprendiz lograra inserto en el proceso productivo.

Sera necesario contar con el total de objetivos de aprendizaje, para tener una visión completa de la formación en la empresa.

c) Validar Plan de Rotación y Puestos de Aprendizajes

Esta acción corresponde con el visto bueno, por parte del gestor de vinculación apoyado por el tutor del plan de rotación y los puestos de aprendizaje que una empresa proporciona para la formación de un educando/aprendiz.

Para lo cual se deberá construir una matriz de correspondencia donde en la trama horizontal se colocarán los resultados de aprendizaje del total de módulos de la carrera correspondiente; en la trama horizontal los objetivos de aprendizaje especificados en cada puesto de aprendizaje del plan de rotación.

\section{CONCLUSIONES}

Para que este modelo funcione, deberían realizarse algunas acciones como: 
- Capacitación de tutores generales y específicos en la modalidad dual.

- Socializar a los estudiantes sobre la normativa vigente.

- Las empresas ofrecerán puestos de trabajo en base al plan de rotación propuesto por los tutores específicos a través de un convenio de formación para los estudiantes que soliciten ser aprendices y cumplan con 150 créditos como requisito.

- Los contenidos del plan de rotación que constan en las áreas específicas serán determinados conjuntamente con: tutor general, tutores específicos y supervisores en coordinación con los representantes de las empresas.

- El tutor específico organiza el aprendizaje y define los objetivos cumpliendo el papel de orientador e integrador que acompaña al estudiante y a la empresa para mantener el lazo entre la experiencia profesional del aprendiz en la empresa y el programa formativo de acuerdo al perfil profesional y relacionado a las líneas de investigación vigentes de la carrera.

- La evaluación y su aprobación es práctica y bajo la responsabilidad de los tutores específicos que cumplen la función adicional de supervisores para que el tutor general otorgue el certificado pertinente.

- Las prácticas pre-profesionales ayudan a elevar la productividad y competencia en la empresa u organización asignada para cumplir con el plan de rotación, en el que se especificará las funciones y los puestos a cubrir realizando un trabajo que cubre un total de 400 horas en dos niveles de 200 horas cada uno.

- Fortalece la vinculación entre la Facultad y la empresa pública y privada.

- Reduce el tiempo de la curva de aprendizaje en el proceso de inducción reduciendo costos de iniciación y reclutamiento en razón de que ya los conocen y es mano de obra calificada.

- Es un sistema que exige la construcción de una adecuada relación entre la Facultad y la empresa, una compleja planeación con docentes capacitados en la modalidad dual para garantizar la oferta hacia los trabajos que se ejecutaran en las empresas como una actividad viable, factible y sostenible.

RECEIVED: April, 2017

REVISED: August, 2017

\section{Referencias Bibliográficas}

ACR. (23 de Agosto de 2016). El Mercurio. Obtenido de https://www.elmercurio.com.ec/551237-formacion-dual-opcion-empresarial/

Admin, X. (17 de mayo de 2014). http://historiapdf.esoftek.com/. Obtenido de http://historiapdf.esoftek.com/fax/ 
Carpi, L. (15 de abril de 2015). http://www.beevoz.com/. Obtenido de http://www.beevoz.com/2015/04/27/historia-del-fax-origen-y-evolucion/

Castillo, A. (2015). Plan de rotacion. Chile.

CERMI. (2008). El modelo social de discapacidad.

Chicaiza, E., \& Parra, R. (2016). http://www.tecnologicosucre.edu.ec/. Obtenido de http://www.tecnologicosucre.edu.ec/carreras/duales.html

Chile, G. d. (2012). http://www.textosescolares.cl/. Obtenido de http://www.textosescolares.cl/usuarios/cpeip/File/2012/MODALIDAD\%20DUAL.p df

E.universitario. (21 de Abril de 2014). http://ecuadoruniversitario.com/. Obtenido de http://ecuadoruniversitario.com/opinion/la-modalidad-dual-es-una-opcion-validaprofesionalizante/

Forero, P. (26 de noviembre de 2012). https://prezi.com. Obtenido de https://prezi.com/xcusvpgap8iu/historia-del-fax/

Francisco H. G. Ferreira, J. M.-C. (2013). La movilidad economica y el crecimiento de la clase media en america latina.

Informatica, m. (25 de julio de 2016). http://www.informaticamoderna.com/. Obtenido de http://www.informaticamoderna.com/Fax_modem_extern.htm

Leiva, J. P. (2005). Las relaciones Ecuador-Estados Unidos.

Leon, K. (3 de mayo de 2009). Google dual . Obtenido de Google dual .

Medina, A. (2006). Plan de Rotacion Empresarial . Ecuador .

Norén, A. (20 de agosto de 2014). http://blog.elsupuesto.com/.

Norén, A. (20 de agosto de 2014). http://blog.elsupuesto.com/. Obtenido de http://blog.elsupuesto.com/nacional/2014/08/formacion-dual/

Pérez, J. (29 de mayo de 2009). http://definicion.de/. Obtenido de http://definicion.de/modem/

Procel, M. (2012). REDACCION DUAL . MEXICO.

Publications, U. N. (2003). Género, previsión y ciudadanía social en América Latina.

Rivero, J. (1999). Educación y exclusión en América Latina.

Rojas, O. (2002). Rotacion Dual . Chile. 
Senescyt. (2015). http://www.senescyt.gob.ec/. Obtenido de

http://www.senescyt.gob.ec/Institutos/formaci\%C3\%B3n-dual.html

Teruel. (2016). http://www.camarateruel.com/. Obtenido de http://www.camarateruel.com/formacion/jornada-formacion-dual/que-es-laformacion-dual.html

Tolley, E. E. (2006). Investigacion Aplicada En.

Valverde, L. (2006). Nodulos Duales. Mexico.



El artículo que se publica es de exclusiva responsabilidad de los autores y no necesariamente reflejan el pensamiento de la Revista Ciencia Digital.

El articulo queda en propiedad de la revista y, por tanto, su publicación parcial y/o total en otro medio tiene que ser autorizado por el director de la Revista Ciencia
